\title{
Robotic Central Pancreatectomy with Pancreaticojejunostomy for Solid Pseudopapillary Neoplasm
}

\author{
Jin Woo Lee, M.D., Juno Yoo, Ji Wool Ko, M.D., Sung Hoon Choi, M.D. \\ Department of Surgery, CHA Bundang Medical Center, CHA University, Seongnam, Korea
}

Purpose: Minimally invasive central pancreatectomy has rarely performed because of its technical difficulty. Robot system enhances surgical dexterity to perform such complex procedures.

Methods: A 29-year-old woman was admitted with acute cholecystitis and an $1.4 \mathrm{~cm}$ enhancing mass was incidentally found at the pancreatic proximal body on computed tomography. Preoperative image studies suggested a neuroendocrine tumor or solid pseudopapillary neoplasm. The patient underwent robotic cholecystectomy and central pancreatectomy with pancreaticojejunostomy.

Results: The total operation time was 280 minutes and the estimated amount of intraoperative bleeding was $100 \mathrm{ml}$. The postoperative recovery was uneventful and she was discharged on the $7^{\text {th }}$ postoperative day. Pathologic examination reported a solid pseudopapillary neoplasm.

Conclusion: The technical difficulties associated with the procedure can be overcome with the help of the wrist-like movement of the robotic instruments, especially for the preservation of splenic vessels and for creating precise anastomoses in narrow spaces.

Keywords: Robotic surgical procedure, Pancreatectomy, Pancreaticojejunostomy, Pancreatic neoplasms

\author{
Received May 16, 2017 \\ Revised May 23, 2017 \\ Accepted May 24, 2017 \\ Corresponding author \\ Sung Hoon Choi \\ Department of Surgery, Bundang \\ CHA Medical Center, CHA \\ University, Cancer Research \\ Building \#524, 59 Yatap-ro, \\ Bundang-gu, Seongnam 13496, \\ Korea \\ Tel: +82-31-780-1824 \\ Fax: +82-31-780-3449 \\ E-mail: feel415@cha.ac.kr
}

Supplementary video file: This article contains supplementary material (https://doi.org/10.7602/ jmis.2017.20.2.74).

This is an Open Access article distributed under the terms of the Creative Commons Attribution Non-Commercial License (http:// creativecommons.org/licenses/by-nc/4.0/) which permits unrestricted non-commercial use, distribution, and reproduction in any medium, provided the original work is properly cited.

Copyright ( 2017 The Journal of Minimally Invasive Surgery. All rights reserved.

\section{INTRODUCTION}

As a parenchyma-sparing surgical procedure, central pancreatectomy $(\mathrm{CP})$ is indicated for benign, borderline malignant neoplasms and isolated metastatic cancer arising at the pancreatic neck and proximal body. ${ }^{1,2}$ However, minimally invasive surgery for $\mathrm{CP}$ has not been actively performed due totherarity of these indications, its technical difficulty, and the potential costs of postoperative morbidity. ${ }^{3}$ Robot surgical systems enhance surgical dexterity to perform such complex procedures.

\section{MATERIALS AND METHODS}

A 29-year-old female was transferred to our hospital with acute cholecystitis. A hypo-attenuated mass of $1.4 \mathrm{~cm}$ in size was incidentally found at the pancreatic proximal body on computed tomography. Magnetic resonance imaging suggested a neuroendocrine tumor or solid pseudopapillary neoplasm (Fig. 1). She was young and the tumor was suspected as being either benign or a borderline malignant neoplasm. Therefore, minimally invasive organ-preserving surgery was regarded as an appropriate approach and so we elected to perform robotic cholecystectomy and CP. 


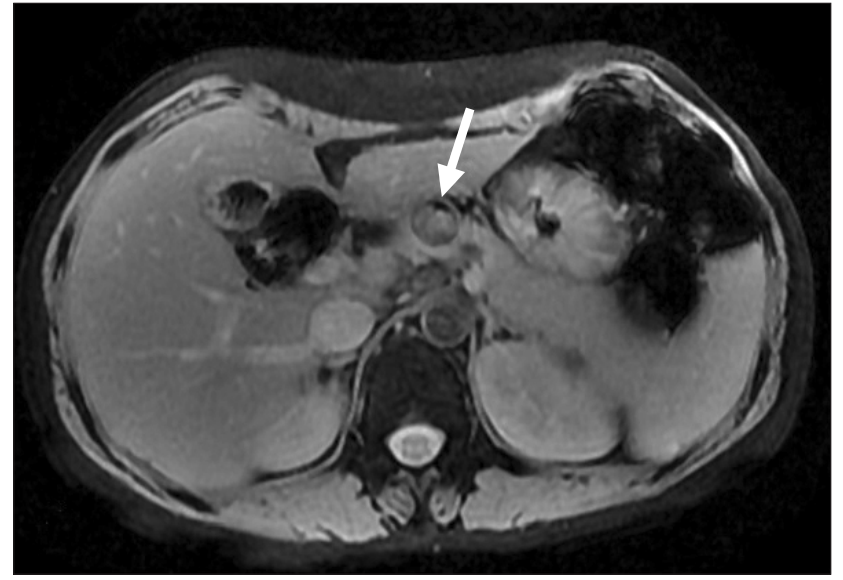

Fig. 1. Magnetic resonance imaging indicated the presence of a neuroendocrine tumor or solid pseudopapillary neoplasm on the proximal pancreatic body, white arrow.

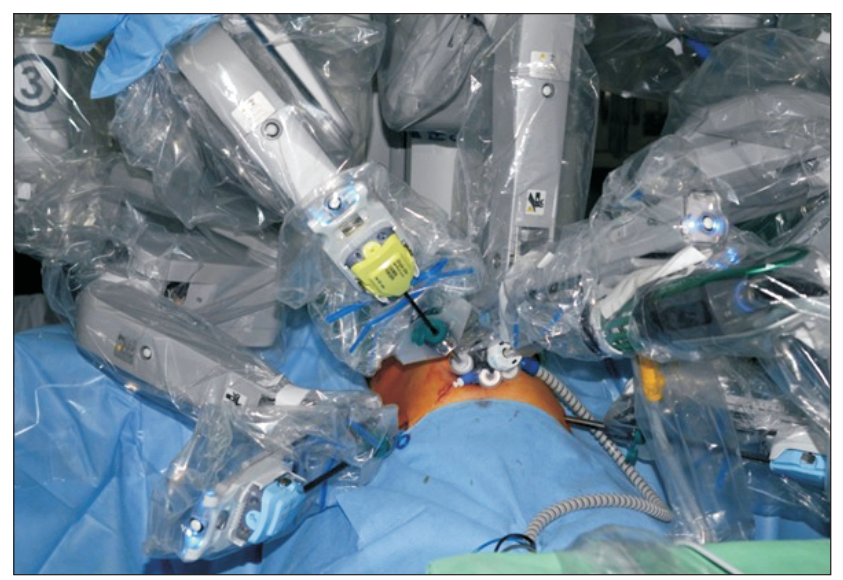

Fig. 2. A glove port with four channels was installed through the umbilicus. Then, a robotic camera and one flexible arm of a single-site system were introduced via the glove port. Two additional trocars for $8 \mathrm{~mm}$ articulating instruments were added bilaterally at a location of $10 \mathrm{~cm}$ apart from the umbilicus.

Her height and weight were $153 \mathrm{~cm}$ and $46 \mathrm{~kg}$ and the diameter of her body at the umbilical level was $28 \mathrm{~cm}$. Her abdominal cavity was too small to apply a conventional robotic multi-arm technique. Then, a reduced-port technique using the da Vinci Si robot surgical system (Intuitive Surgical, Sunnyvale, CA) was designed. The Glove port with four channels (NELIS, Bucheon, Gyonggi-do, Korea) was installed through the umbilicus. ${ }^{4}$ A $12 \mathrm{~mm}$ camera and one flexible arm of the single-site system were introduced via the Glove port (Fig. 2). Two additional $8 \mathrm{~mm}$ trocars for articulating instruments and placed bilaterally $10 \mathrm{~cm}$ apart from the umbilicus (Fig. 3). Cholecystectomy was performed in a conventional

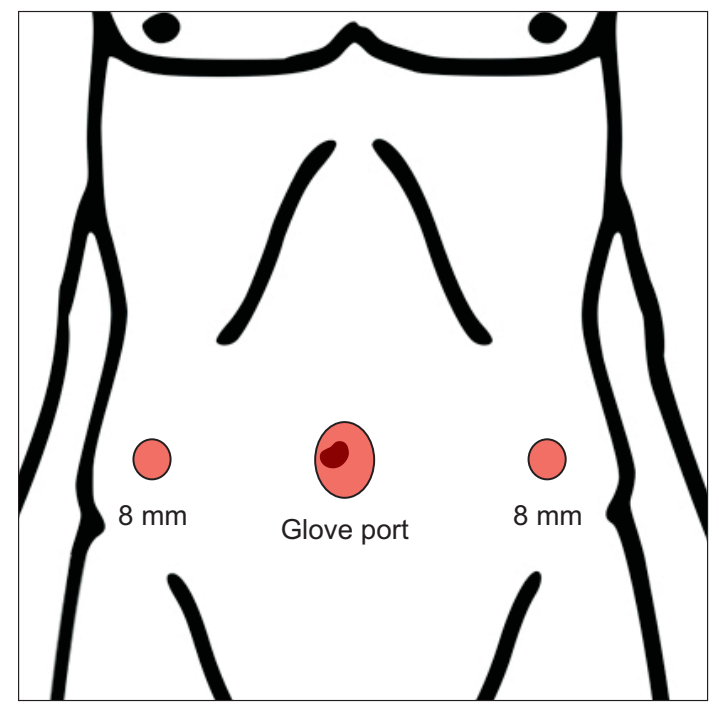

Fig. 3. Port placement for this reduced-port system.

manner. Following retraction of the stomach using the singlesite arm, the pancreatic neck and proximal body were prepared for segmental resection preserving the splenic vessels. The pancreatic neck was resected using a $60 \mathrm{~mm}$ medium thick endoGIA and the distal segment of the pancreas was transected using ultrasonic shears. The remnant distal pancreas was reconstructed using pancreaticojejunostomy with a two-layered and duct-to-mucosa technique. The specimen was retrieved through the umbilical wound and a drain tube was inserted through the left $8 \mathrm{~mm}$ trocar site.

\section{RESULTS}

The total operation time was 280 minutes and the estimated amount of intraoperative bleeding was $100 \mathrm{ml}$. Pathological assessment indicated that the mass was a solid pseudopapillary neoplasm that was $1.4 \mathrm{~cm}$ in size, which was removed using a surgical resection margin of $1.0 \mathrm{~cm}$ (Fig. 4). Five tumor free lymph nodes were obtained and neither lymphovascular invasion nor perineural invasion were identified. The postoperative recovery was uneventful and she was discharged on the 7th postoperative day in good condition. She undergoes follow-up on a routine basis for surveillance without adjuvant therapy.

\section{DISCUSSION}

Although $\mathrm{CP}$ is an appealing surgical option for treating benign or borderline malignant neoplasms of the pancreatic neck or proximal body of pancreas, a minimally invasive approach has been rarely attempted., Laparoscopic distal pancreatectomy is becoming the standard procedure for left-side 


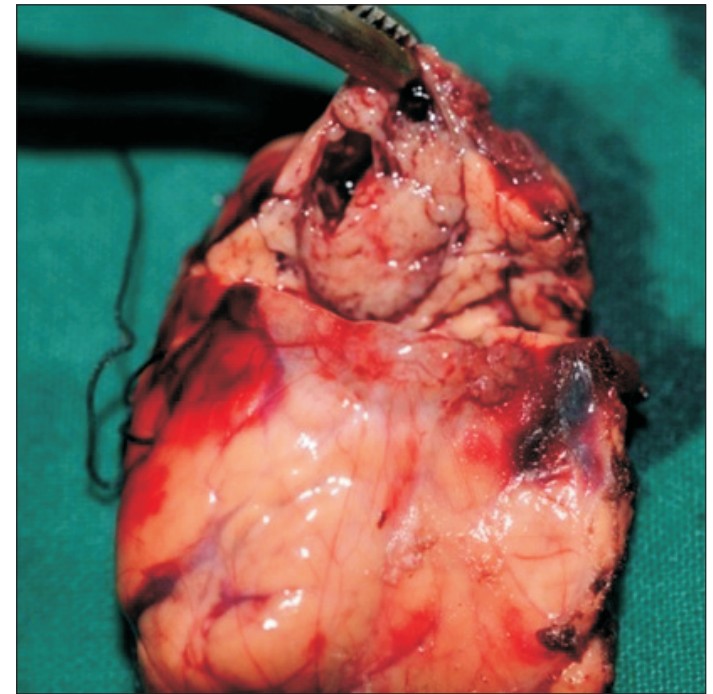

Fig. 4. Surgical specimen obtained from a segment of the proximal body including the tumor.

pancreatic diseases ${ }^{7}$ and the use of laparoscopic pancreaticoduodenectomy has been actively pursued in recent years. ${ }^{8} \mathrm{De}^{-}$ spite the reduced extent of the surgical field in $\mathrm{CP}$ compared to distal pancreatectomy and pancreaticoduodenectomy, the use of two surgical planes in $\mathrm{CP}$ is a major obstacle for the laparoscopic approach. In this technique, the main dissection plane is the pancreatic neck, while the reconstruction plane is changed to a position that is distal to the pancreatic neck since a segment of the proximal pancreatic body is removed. In laparoscopic surgery, the limited movement of the non-articulating instruments could be overcome via the exact placement of ports toward the target organ. ${ }^{9}$ Therefore, changes in the surgical plane complicate the proper placement of the laparoscopic instruments to the target organs. This is the major reason why laparoscopic central pancreatectomy has not been actively performed. However, this change in the target surgical plane is not a serious obstacle for robotic systems given the freedom in angulation of the 'endowrist' movement enabled by robotic instruments. This is a meaningful advantage of using a robotic approach in $\mathrm{CP}^{10}$

For this operation, we used a 'reduced-port system.' The reduced-port system has value not only in reducing the $\mathrm{se}^{-}$ verity of wounds, but also in avoiding a collision between the robotic arms in the narrow abdominal cavity of some patients. We used a glove port in the umbilicus to introduce a robotic camera and a single-site arm through the port, which enabled access of assistant instruments, including a suction catheter, laparoscopic ultrasonography, and laparoscopic surgical stapler. The single-site arm was used for traction or retraction, including of the stomach and pancreas. The two articulating instruments worked effectively to perform the fine dissection of splenic vessels and a delicate anastomosis of pancreaticojejunostomy.

$\mathrm{CP}$ is optimal removing benign or borderline malignant tumors while minimizing the loss of the endocrine and exocrine function of the pancreas. Although its technical difficulty and the potential costs of postoperative complications by the two cut surfaces of pancreas, $\mathrm{CP}$ is greatly facilitated by the introduction of robotic surgical systems. The technical difficulties associated with the procedure can be overcome with the help of the wrist-like movement of the robotic instruments. Further experience with and studies of the technical generalization or actual benefits of robotic $\mathrm{CP}$ are necessary.

\section{REFERENCES}

1) Muller MW, Friess H, Kleeff J, et al. Middle segmental pancreatic resection: An option to treat benign pancreatic body lesions. Ann Surg 2006;244:909-918; discussion 918-920.

2) Crippa S, Bassi C, Warshaw AL, et al. Middle pancreatectomy: indications, short- and long-term operative outcomes. Ann Surg 2007;246:69-76.

3) Sauvanet A, Partensky C, Sastre B, et al. Medial pancreatectomy: a multi-institutional retrospective study of 53 patients by the French Pancreas Club. Surgery 2002;132:836-843.

4) Choi SH, Hwang HK, Kang CM, Lee WJ. Transumbilical single port laparoscopic adrenalectomy: a technical report on right and left adrenalectomy using the glove port. Yonsei Med J 2012;53:442-445.

5) Sa Cunha A, Rault A, Beau C, Collet D, Masson B. Laparoscopic central pancreatectomy: single institution experience of 6 patients. Surgery 2007;142:405-409.

6) Rotellar F, Pardo F, Montiel C, et al. Totally laparoscopic Rouxen-Y duct-to-mucosa pancreaticojejunostomy after middle pancreatectomy: a consecutive nine-case series at a single institution. Ann Surg 2008;247:938-944.

7) Mehrabi A, Hafezi M, Arvin J, et al. A systematic review and meta-analysis of laparoscopic versus open distal pancreatectomy for benign and malignant lesions of the pancreas: it's time to randomize. Surgery 2015;157:45-55.

8) Dai R, Turley RS, Blazer DG. Contemporary review of minimally invasive pancreaticoduodenectomy. World J Gastrointest Surg 2016;8:784-791.

9) Oppenheimer P, Weghorst S, Williams L, et al. Laparoscopic surgical simulator and port placement study. Stud Health Technol Inform 2000;70:233-235.

10) Kang CM, Kim DH, Lee WJ, Chi HS. Initial experiences using robot-assisted central pancreatectomy with pancreaticogastrostomy: a potential way to advanced laparoscopic pancreatectomy. Surg Endosc 2011;25:1101-1106. 